\title{
WEB PERFORMANCE PREDICTION USING GEO-STATISTICAL METHOD
}

\author{
P. Lakshmanan ${ }^{1}$, N. Sangeetha ${ }^{2}$ \\ ${ }^{1}$ P.G Student, ${ }^{2}$ Asst Professor, M.E CSE, Alpha college of Engg, Chennai, T.N, India
}

\begin{abstract}
To provide Qos in distributed web systems, it is necessary to perform predicting in web systems. Then provide prediction technique on time and space of web resource downloaded using turning bands geostatistical simulation method. Web resources are obtained from a group of web servers worldwide by multi agent measurement systems which continuously monitor the web servers located in different geographical localization. The outcome of high-quality of Web performance calculation completed by resources of the Turning bands method, particularly in the situation when we place the Web servers were monitored by a Multi-agent Measurement Systems $(M W I N G)$ localized in Poland. By means of satellite navigation system real life data like accident cases, first aid rescuers can accurately locate the place within certain specified range with maximum error control, so that the injured people can be saved as soon as possible. This method will save time or the areas in the outer part of the main zone.
\end{abstract}

Keywords-Vibration sensor, Quality of Service, Networked Distributed System.

\section{INTRODUCTION}

New perception of distributed computer systems (DCSs) include, among others networked control systems (NCSs) and service-oriented architectures, Describe general architecture, best practices, and current contributions known under the umbrella terms Internet of Things (IOT), and Web of Things (WOT) then the Web ecosystem will be a desirable medium for networked platforms for smart things (e.g., sensor networks and embedded devices), as well as for industrial and manufacturing systems. The foundation to improve the bestachievement traffic rather than real-time traffic, DCSs developed in the Internet have to deal with challenging communication issues, because of these systems networking requirements following the real-time mode of operation. The most important requirements concern performance, reliability, quality of service (QOS), and security. To kind of the process more predictable, Web information should be DCSs is need a spatial-temporal Web performance forecasting, where the temporal prediction addresses the forecasting of $\mathrm{Web}$ performance is calculating ordered by one or more dimensions of time (e.g., the minute-by-minute, hour by-hour, and day-byday).

The spatial prediction concerns predicting of the performance behavior across nodes of DCS (more specifically, between Web clients and Web servers).The predicted performance can be used in selection of the best performance Web server and best in space and in time. The first is the introduction of a new spatial and temporal methodological approach to the routine forecast of distributed computer systems, recognized on the theory and application of geostatic. The second is a Web performance prediction system based on the widely proven
Turing band method, which gives efficient and accurate forecasting, as well as reliable and exact results. Then third impact is that analysis uses real-life data collections gathered for various clients (agents) monitoring many Web servers localized in different Internet geographic locations.

From the reports of data analysis, it can draw this conclusion some extremely vicious incident cannot be rescued, but some passing a way of life in an accident can be redemptive, but due to timing delays in the rescue of personnel caused the casualties. Vibration sensors are applied in a quantity of requests to measure acceleration and/or vibrational activity. Vibration sensors can be operated to regulate whether the machinery is operating correctly. Vibration sensors can be useful for checking the situation of rotating machinery, where warmness or excessive vibration could specify excessive loading, insufficient lubrication, or bearing wear.

\subsection{Network Control Systems}

A Networked Control System (NCS) is a control circles are closed through a real-time system. The defining eye of an NCS is that control and response indications are swapped between the system's workings in the form of material packages through a system.

Intermediate nodes are mesh nodes that are involved in constructing the hierarchical multicast architecture, i.e., it include the mesh nodes within an access area, irrespective of whether or not it become active forwarders on multicast traffic distribution trees. 
Unique characteristics of a Network Control Systems are,

- Sensors to get material

- Supervisors to deliver choice and instructions

- Actuators to achieve the control instructions and Message network to permit argument of material.

\section{RELATED WORK AND EXISTING MODEL}

Sensor Networks newly take to originate into importance outstanding to the detail that it have the possible to change several sections of our economic organization and life - from transport, ecological screen, passage and healthcare industries. Carrying the material group and message plays a key role in Intellectual Conveyance System. Inappropriately, most predictable ITSs can only sense the automobile in a permanent location and the communication chains and power cables raise the cost of creation and conservation. Since of the benefits of the wireless sensor network (WSN) such as little power intake, wireless delivery, and suppleness without cable limitations. The practice of WSN in ITSs is predictable to be intelligent to overcome the overhead problems. Such systems are repeatedly installed in supply-controlled surroundings, for occurrence with battery functioned protuberances running released. These restraints prescription that sensor network difficulties are the finest loomed in a all-inclusive method, by mutually seeing the corporeal, networking, and application layers and creation main enterprise skill-offs diagonally the coatings.

The chief impartial of WSNs is to observer physical or ecological phenomena like hotness, sound, shaking, comparative clamminess, impurities, etc. It also gathers facts to be stated to a dominant dispensation component that examines the collected statistics and income positive methods consequently.

An intelligent traffic system (ITS) usages modern skills like communications and information knowledge for circulation flow organization. It also inspires automobile motorists to use substitute procedures of transportation, and delivers material for driving carefully, for circumventing traffic roadblocks. This application assistances in traffic blocking, accidents and the impression subsequently that it generate and also it supports in saving time and more significant the drivers/travelers have reconciliation of mind while it are peripatetic. The explanation is Wireless Sensor Network to be positioned. Vehicular Ad-hoc network is similarly mentioned to as vehicle-to-vehicle statement. Since at this time the nodes or routers are the automobiles i.e. cars, motorbikes, Lorries, buses etc. It means that the undertaking of the protuberance is profitable to be controlled by the features like road progression, transportation jams or traffic instructions and principles. If this materializes that the node is motionless/ limited then VANET has to be positioned in a permanent surroundings. This permanent network can be organized on that positions which are dangerous and glowing known for risky weather conditions, dangerous intersections etc.
The Intelligent Transportation System is that which exploits the indication knowledge and Communication can be a complete method. It has a portion of upcoming that can inspire the depositors to exertion concluded it and style the wandering additional sheltered and safe. Particular of the submissions of ITS, are declared under which also can be used in upcoming after applying VANETs in Pakistan:

- Involuntary highway implementation complete one-to-one care

- Adjustable speed restrictions at dissimilar conditions

- Energetic Traffic Light Arrangement

It is a hopeful and developing wireless communication skill to progress road protection. It suggests an outline and the necessities of VANET submissions are occupied into interpretation. Then similarly instruction several permitting equipment for the enterprise agenda. It has confidence in that is study can deliver a recommendation for the plan of a VANET Network to be arrayed in Pakistan. This will assistance drifting to be safe as there will be caution that will be producing done IHVW system whenever several events occurs. There are commonly two ways of explanation of complications produced due to the damaged presentation of the Network. The first is making enhancements in the quality of communication protocols, including the development of real-time protocols, protocol tuning, as well as upgrading existing network technologies to support needed communication requirements.

\section{PROPOSED SYSTEM}

Proposed system use to improve the web performance and service oriented architecture to increasing the quality of service using Turning band. Then propose to use the TB geostatistical method to forecast spatio-temporal web performance. This method considers the existence of a functional relationship between what happens at one geographical location and what happens elsewhere. The TB method uses a geostatistical technique for interpolation of the studied parameter at an unmonitored location from available observations at nearby locations. Thus, this method gives as results not only forecasted performance for studied Web servers, but also for the neighborhoods. The forecasting results for locations where there are no servers is just a simulation not a factual data. Nevertheless, it could be helpful, for example, to optimize traffic in the network or to suggest adding new servers in analyzed locations. This method was also chosen because it requires a minimum amount of input data to perform forecasting. A vibration sensor is fixed in the Motor vehicle. If several accidents happen to the vehicle, nearly the vibration would be originated; the vibration instrument will be activated. 


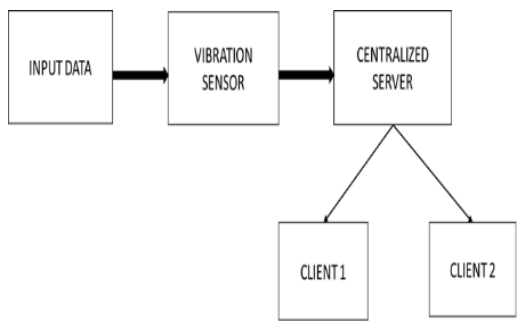

Fig: System Design

The vibration instrument will wait for certain period for the motorist to press the button which is assumed in obverse of the motorist for certain shuddering. If driver presses the button it is understandable that no fatal accident has occurred, because of the driver consciousness. But no input is given by the driver for the period of time, the Vibration sensor determination activate the GPS, GPS which is tailored in the vehicle resolve now twitch communicate through the satellite broadcasting, and become the Longitude and Latitude Standards. Dual caring the ideals are approved to the Centralized server. The centralized server will need the set of record, which encloses all the Clinic's Longitude and Latitude Ideals.

\section{SYSTEM DESCRIPTION}

\subsection{Turning Bands Method for Centralized Server}

After the time sequence, if no input of pressing switch event is occurred, then system will consider that accident has occurred, so immediately GPS to fetch the exact location of the place where the accident has occurred. This GPS value is sent to the centralized server by J2ME Mobile tool kit wirelessly to Identification \& Tracking the Location of Vehicle \& Wireless Communication by J2ME to the Central Server. The Installation Instructions for the Java Wireless Toolkit If you do not have the Java Development Kit (JDK) or only have the Java Runtime Environment (JRE) installed, install the latest JDK Present steady issue of Java is JDK 6 Apprise 7 but squared the web page in situation there are fresher non-beta issues obtainable. Then following transfer the Java Wireless Toolkit (previously called J2ME Wireless Toolkit) Track the installer (for sample, for Windows it is sun_java_wireless_toolkit-2_5_2-windows.exe). The installer payments whether a well-matched Java atmosphere has remained pre-fitted. J2ME frameworks that is appropriate for dissimilar market sections - CLDC for minor devices and CDC for superior devices.

\subsection{Identifying Client Using Hospital Server}

After receiving of the Location information by the centralized server, it computes the Shortest Path algorithm to find out the nearest Hospital by comparing with the Database. This Data base will have all the Hospital Server's Longitude \& Latitude Values. The Accident zone's Longitude \& Latitude is compared with the Longitude \& Latitude of entire Hospitals.
Shortest path algorithm is applied to fetch the nearest hospital. The Hospital server, Google Earth is installed. This Hospital server is set up in the each \& every Hospital to fetch out the exact location of accident occurred place. Google Earth is a simulated globe, map and environmental material sequencer that were initially termed Earth Watcher 3D and stood shaped by Aperture. It maps the Soil by the superimposition of pictures acquired from satellite descriptions, airborne camerawork and GIS 3D sphere. Creating content in Google Earth enables a user to create personal Google Earth files and while this is not exceptionally difficult, there are some specifics that you need to know the interface Information in Google Earth is presented in place marks. These are the pushpins or other icons that you click on when you are using Google Earth. Clicking on a place mark opens a place mark window, and displays the associated content.

\subsection{Vibration Detection \& Avoidance of False Prompting}

Vibration Sensor is fitted in the Vehicle, once the accidents occurs; Vibration sensor identifies the accident, immediately triggers the Alarm unit for the alerting the driver. Switch unit is provided to the driver to stop the communication. If any minor accident occurs during driving, driver can control the communication by pressing the switch. This stops the information routing to the centralized server. Vibration sensors are utilized in a number of applications to measure acceleration and/or vibration activity. Vibration sensors container be applied to control whether the equipment is working correctly. Vibration sensors can be valuable for observing the state of revolving equipment; anywhere warmness or extreme vibration might designate extreme loading, insufficient lubrication, or manner attire. Such sensors are likewise used in geophysical requests and submissions demanding accelerometers. Vibration sensors stand used as collision sensors in interior burning appliances. In instruction to assurance that a device is operative under best conditions, it is essential to exactly monitor its real operating public.

Unique device familiar to be extremely useful for this determination is the device vibration sensor. Vibration or shockwave sensors are normally used in alarm systems to start an alarm whenever the plans to which it is involved are touched, moved, or else vibrated.

\subsection{Turning Bands Method for Identifying Nearest}

\section{Service}

Identification of exact location of accident place in the nearest hospital server and rescue process it getting once information of Longitude \& Latitude values of the accident occurred is transmitted to the nearest Hospital server. In the Hospital server, the Google earth is started dynamically to show out the 
exact place of Accident Place. Google Earth software identifies the exact place by converting the Longitude \& Latitude values into KML file format to dynamically open the Google Earth to point not the exact location. Once Nearest Hospital identifies the exact location of the accident place, it immediately send the ambulance to rescue the people who met with accident. Latitude it should be quantity of the pointed distance on a highpoint north or south of the equator. Latitudinal appearances run flat around the earth in similar concentric positions from the equator to individually of the poles. Longitude it must be the quantity of the angular distance on a height east or west of the prime meridian. Longitudinal appearances spread from the north to South Pole nearby the earth, like parts of an orange. Horizontal group method will be labels the technique used to control the latitude and longitude organizes for a fact on the earth. This stipulates what kind of way or method was used to recognize the latitude and longitude, e.g., an address, an intersection, a Global Positioning System (GPS) method, a survey block centric, etc.

\section{CONCLUSIONS}

The implementation of the k-nearest node (KNN) algorithm, the Turning band algorithm to link interruption recovery. Each package is verified separately at the time of enlargement by means of the information's and has established that this program related together in the technique stated in the agendas requirement, the computer structure and its location is verified to the fulfillment of the operator. The system that has been established is acknowledged and showed to be acceptable for the manipulator. And so the system is profitable to be applied very shortly. A humble functioning process is involved so that the handler can understand the dissimilar purposes noticeably and quickly. Initially as a first step the executable form of the application is to be created and loaded in the common server machine which is accessible to the entire user and the server is to be connected to a network. The final stage is to document the entire system which provides components and the operating procedures of the system. Then the Vibration sensor will activate the GPS, GPS which is tailored in the automobile will currently twitch interconnect with the satellite, and get the Longitude and Latitude Standards. Dual caring the ideals are agreed to the Centralized server. The centralized server will be having the usual of record, which covers all the Hospital's Longitude and Latitude Standards. The vehicle's Longitude and Latitude Standards are likened with the Longitude and Latitude Ethics of the Hospital, to find out the nearest Hospital to the accident zone.

\section{REFERENCES}

[1]. Alison K. Brown, Ph.D.,'Test Results Of a GPS/Inertial Navigation System Using a Low Cost MemsImu,"Aerospace and Electronic Systems Magazine, IEEE (Volume: 20, Issue: 9).

[2]. A. Onat, T. Naskali, E. Parlakay, and O. Mutluer, "Control over imperfect networks:Model-based predictive networked control systems," IEEE Trans. Ind. Electron., vol. 58, no. 3, pp. 905-913, Mar. 2011.

[3]. Cetin Mekik and Murat Arslanoglu, "Investigation on Accuracies of Real Time Kinematic GPS for GIS Applications," ZonguldakKaraelmas University, Engineering Faculty, Dept. of Geodesy and Photogrammetry Eng., Zonguldak, Turkey.

[4]. Franke,H.A, "Grid-M: Middleware to Integrate Mobile Devices, Sensors and Grid Computing,'Fed. Univ. of Santa Catarina, Florianopolis; Koch, F.L.; Rolim, Westphall, C.B.

[5]. Qingyuan Li, Deguo Su, Hongsheng Li, Haochen Liu, Lijian Sun, "Approach To General Data Model Of GIS Symbol Library And Symbol Library Data Exchange Xml Schema," Geo-spatial Information Science 04/2011.

[6]. Q. Wang and S. Gopalakrishnan, "Adapting a mainstream Internet switch architecture for multihop real-time industrial networks," IEEE Trans. Ind. Inform., vol. 6, no. 3, pp. 393404, Aug. 2010.

[7]. Rahat Ali Khan, Shakeel Ahmed Shah,Muhammad Abdul Aleem,Zulfiqar Ali Bhutto,Asad," Wireless Sensor Networks a Solution for Smart Transportations,'SS.B., Electrical Science and Engineering (1999) Massachusetts Institute of Technology.

[8]. Shuang-Hua Yang, "Internet-based Control Systems: Design and Applications, Advances in Industrial Control, S-H. Yang, Ed. London, U.K.: Springer-Verlag, 2011.

[9]. Xianlin, Huang, Guofeng, Wang,'Design and application of single-antenna GPS/accelerometers attitude determination system,"Systems Engineering and Electronics, Journal of (Volume: 19, Issue: 2). 